\title{
Impact perforation of sandwich panels with graded hollow sphere cores: Numerical and Analytical investigations
}

\author{
Ibrahim Elnasri ${ }^{1,2^{*}}$ \\ ${ }^{1}$ Laboratoire de Génie Mécanique, Ecole Nationale d'Ingénieurs de Monastir, University of \\ Monastir, Av. Ibn ElJazzar, Monastir - 5019, Tunisia \\ ${ }^{2}$ Faculty of Engineering, University of Tabuk, P.O. Box 741, Kingdom of Saudi Arabia
}

\begin{abstract}
In this study, we numerically and analytically investigate the impact perforation of sandwich panels made of $0.8 \mathrm{~mm} 2024-\mathrm{T} 3$ aluminum alloy skin sheets and graded polymeric hollow sphere cores with four different gradient profiles. A suitable numerical model was conducted using the LS-DYNA code, calibrated with an inverse perforation test, instrumented with a Hopkinson bar, and validated using experimental data from the literature. Moreover, the effect of boundary conditions on the perforation resistance of the studied graded core sandwich panels was discussed. The simulation results showed that the piercing forcedisplacement response of the graded core sandwich panels is affected by the core density gradient profiles. Besides, the energy absorption capability can be effectively enhanced by modifying the arrangement of the core layers with un-clumping boundary conditions in the graded core sandwich panel, which is rather too hard to achieve with clumping boundary conditions. Finally, an analytical model, taken account only gradient in the quasi-static plateau stress, is developed to predict the top skin pic peak load of the graded sandwich panel.
\end{abstract}

\section{Introduction}

One of the most important problems in sandwich panels is the damage that occurs as a result of the discontinuity of the mechanical properties at the boundary between the face sheets and the core. When these sandwiches are exposed to impact loading, for example, the shear stresses can cause the face sheets to detach from the core. Over the past few decades, functionally graded material (FGM) cores have increasingly been used in sandwich panels because of their ability to reduce thermal and residual stresses induced between the face sheets and core material in comparison to conventional sandwich panels [1-2]. Various numerical and experimental studies have been performed to investigate the impact response of functionally graded foam sandwich panels. However, there has been no consensus regarding whether graded core sandwich panels have better perforation resistance than that

\footnotetext{
* Corresponding author: ibrahim.nasri@ @issig.rnu.tn
} 
of ungraded core sandwich panels with equal mass [3-8]. The aim of this study is to provide insights into the impact perforation of sandwich panels made of $0.8 \mathrm{~mm} 2024-\mathrm{T} 3$ aluminum alloy skin sheets and graded polymeric hollow sphere cores. Four gradient profiles were taken as a reference: A-4321, B-1234, D-2431, and E-4123. The four numbers constituting the names of these profiles signify the sequence of the layer's density beginning from the top skin. A-4321 (B-1234) signify then linearity decreasing (increasing) graded hollow spheres cores. The D-2431 gradient profile is a profile with an unbalanced V-shape with a few dense ends, whereas the E-4123 gradient profile has an unbalanced V-shape with more dense ends.

\section{Problem statement}

In this numerical study, sandwich panels with a diameter of $60 \mathrm{~mm}$, made of two $0.8 \mathrm{~mm}$ 2024-T3 aluminum alloy skin sheets and a core with polymeric hollow spheres of $40 \mathrm{~mm}$, were used to model the impact perforation of sandwich panels with FGMs. Table 1 lists the basic characteristics of the graded core sandwich samples. Gradient profiles were obtained by changing the hollow spheres' density every $10 \mathrm{~mm}$. The density of the layers was considered to be in the range of 156 to $468 \mathrm{~kg} / \mathrm{m}^{3}$.

Table 1. Setting Basic characteristics of the graded core sandwich samples

\begin{tabular}{|c|c|c|c|c|c|}
\hline $\begin{array}{c}\text { Reference } \\
\text { Sandwich } \\
\text { (kg/m3) }\end{array}$ & $\begin{array}{c}\text { Core } \\
\text { density }\end{array}$ & $\begin{array}{c}\text { First layer } \\
\text { density }\end{array}$ & $\begin{array}{c}\text { Second } \\
\text { layer } \\
\text { density }\end{array}$ & $\begin{array}{c}\text { Third layer } \\
\text { density }\end{array}$ & $\begin{array}{c}\text { Fourth } \\
\text { layer } \\
\text { density }\end{array}$ \\
\hline A4321 (sandwich) & 302 & 468 & 343 & 242 & 156 \\
\hline B1234 (sandwich) & 302 & 156 & 242 & 343 & 468 \\
\hline D2431 (sandwich) & 302 & 343 & 468 & 242 & 156 \\
\hline E4123 (sandwich) & 302 & 468 & 156 & 343 & 242 \\
\hline
\end{tabular}

To validate the numerical simulation results, the density of each layer was chosen to match that of the sample in the experimental research performed by Zeng et al. [8]. In that study, no experimental results were obtained for the impact perforation of such graded core sandwich panels with intermediary gradient profiles: D-2431 and E-4123. An inverse perforation technique with Hopkinson pressure bars was used to study graded core sandwich panels under impact loading. An impact velocity of $45 \mathrm{~m} / \mathrm{s}$ was used in all tests.

\section{Finite element modelling}

In this study, we developed a numerical model of the perforation of sandwich panels with graded cores using the LS-DYNA code. The model consisted of two aluminum skin sheets and a graded core, with the core comprising four layers with different densities. As shown in Fig. 1, the model consisted of nine parts. Part 1 is represented as an elastic type 1 material. Part 9 and Part 2 are modeled as rigid bodies with type 20 material. The aluminum sheets are modeled by shell elements with five integration points in thickness, and the four graded layers are modeled using brick elements. The sandwich panels were modeled with a fine mesh in the penetration area just under the perforator. Shell elements with dimensions of $0.467 \times$ $0.467 \mathrm{~mm}$ were used for the aluminum skin sheets. Each layer was modeled using brick elements with dimensions of $0.467 \times 0.467 \times 0.666 \mathrm{~mm}$. Tie constraints were applied between the top skin and the first layer (Part 4) and between the bottom skin and the last layer (Part 7). Contact between the Split Hopkinson pressure perforator bar and the sandwich panels was defined using eroding surface-to-surface contact. All z-translational and zrotational degrees of freedom in the no-loading end of the Hopkinson bar were fixed. 


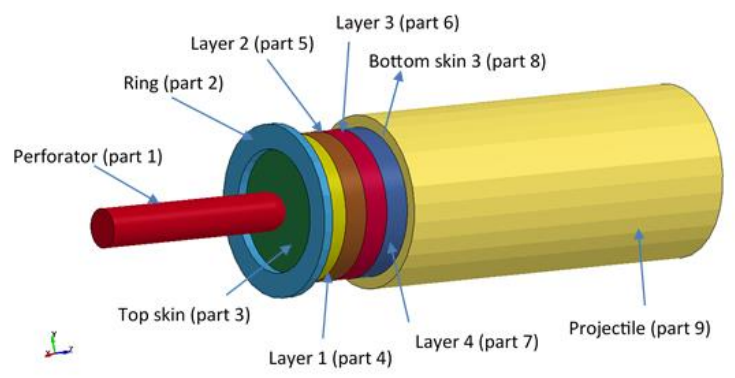

Fig. 1. Numerical model for the perforation of a graded core sandwich panel, testing configuration.

The velocities of the sample and the bullet were modeled and input as initial velocity nodes. The behavior of the skin sheet was modeled using the constitutive material as a type 104 model proposed by Lemaitre [9]. The parameters used for the 2024-T3 aluminum sheets were identified from the normalized tension tests by Pattofatto et al. [10].The four layers with different densities were represented by a simple material model *MAT_HONEYCOMB. Curves of normal stress versus volumetric strain for different density foams were obtained from the quasi-static compression tests performed by Zeng et al. [11].

\subsection{Validation of the numerical simulation}

Using the aforementioned constitutive models, a simulation of a piercing test of sandwich panels with A-4321 gradient profile at $45 \mathrm{~m} / \mathrm{s}$ was performed first to validate the numerical approach with the experimental results obtained by Zeng et al. [8]. Figure 2 compares the curves of the impact force versus displacement obtained from both the experiment and simulation for graded core sandwich panels with a decreasing gradient profile (A-4321). The numerical model predicts the peak forces for the perforation of the top and bottom skins with a high degree of accuracy in comparison to the measured piercing forces. According to the results, we believe that the predictability of the proposed numerical approach is reliable in general.

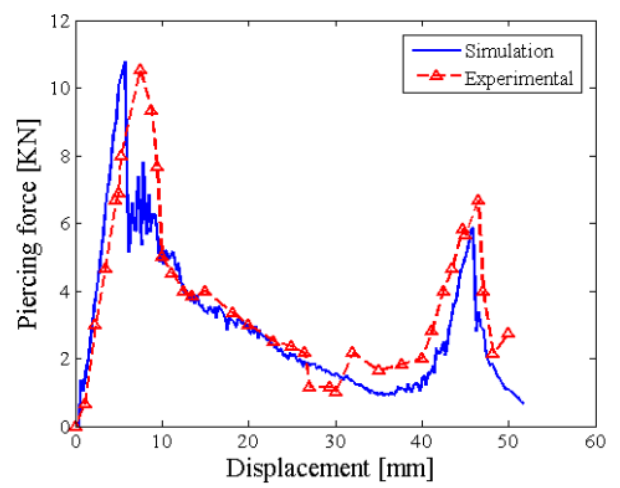

Fig. 2. Plots of piercing force versus displacement of graded core sandwich panels. Comparison between the simulation and experimental results [8].

\subsection{Impact perforation results}

All the force-displacement curves of all graded core sandwich panels are shown in Fig. 3. It was found that the force-displacement response is affected by the core density gradient. More 
specifically, A-4321 has the highest incident piercing top skin peak force, followed by E4123, D-2431, and B-1234, when comparing the peak force for the perforation of the top skin sheet. On the other hand, the piercing bottom skin peak force, which is independent of the core density, has the same peak force value for all graded core sandwich panels, which is presumed to be the piercing force for single skin sheets. These results indicate that the gradient profiles significantly affect the piercing force-displacement curves of graded core layers.

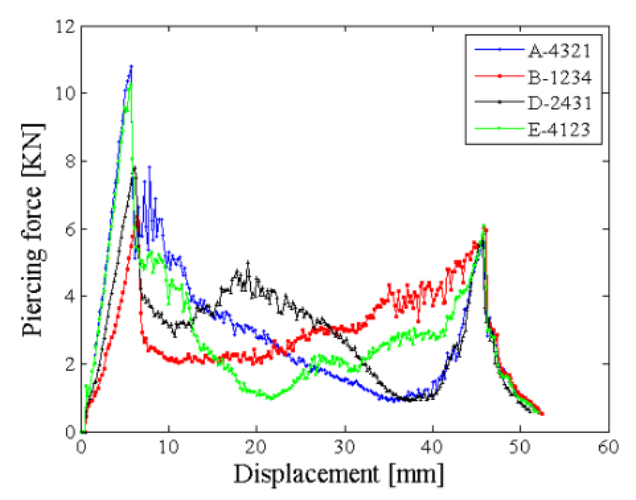

Fig. 3. Plots of simulated piercing force versus displacement $(\mathrm{v}=45 \mathrm{~m} / \mathrm{s})$.

\subsection{Effect of the boundary conditions}

To investigate the effect of the boundary conditions on the response of such graded core sandwich panels, impact perforation without integrating a ring in the numerical approach was performed. For the sake of clarity, this numerical approach is called simulation with unclumping boundary conditions, whereas the first numerical approach is called simulation with clumping conditions (with a ring). The results are shown in Fig. 4 in terms of the energy absorbed versus displacement curves for these virtual impact perforation tests.

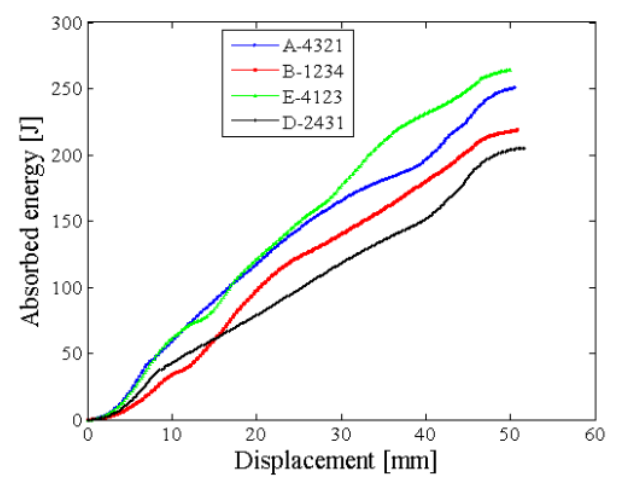

Fig. 4. Plots of absorbed energy versus displacement under impact loading $(v=45 \mathrm{~m} / \mathrm{s})$.

An enhancement of the energy absorbed was observed in comparison to the results with clumping boundary conditions. Table 2 summarizes the predicted values of absorbed energy for both numerical approaches. The results indicate that the best strategy to enhance the energy absorption is to set un-clumping boundary conditions for the fixed sandwich system and to place the higher layer in contact with the top skin followed by the weaker layer as a second layer (i.e., graded sandwich panel with the gradient profile E-4123). These results 
suggest that the effect of the layer arrangement would be significant only if the boundary conditions of the graded core sandwich panels were of the un-clumping type.

Table 2. Prediction of the energy absorption of the sandwich with the both numerical approaches.

\begin{tabular}{|l|c|c|c|}
\hline Sandwichs & $\begin{array}{c}\text { Energy absorbed (J) } \\
\text { clumping conditions }\end{array}$ & $\begin{array}{c}\text { Energy absorbed (J) } \\
\text { Un-clumping conditions }\end{array}$ & $\begin{array}{c}\text { Enhancement } \\
\text { \% }\end{array}$ \\
\hline A-4321 & 154.1 & 250.5 & 62.5 \\
\hline B-1234 & 152.1 & 218.6 & 43.7 \\
\hline D-2431 & 151.4 & 205.2 & 35.58 \\
\hline E-4123 & 149.2 & 264.2 & 77.1 \\
\hline
\end{tabular}

\section{Analytical Model}

Based on the rigid perfectly plastic locking (RPPL) shock model [12], a simple analytical model can be derived. The simple model can be deduced from the impact piercing force model from the impact perforation of the sandwich panel based aluminium Cymat foam reported by Elnasri et al. [13] by introducing a gradient profile in terms of the quasi-static stress plateau. The impact force can be then be written as [13]:

$$
\begin{aligned}
& F_{P}=\sqrt{2} A \pi D_{C}\left(\varepsilon_{c r d f}\right)^{1.5}+\pi D_{C} \times 550.8 \times 10^{3}\left(\frac{\rho_{0}}{\rho_{s}}\right)^{1.8}+\pi \frac{D_{C}^{2}}{4} K \sigma_{p l} \\
& +\pi \frac{D_{C}^{2}}{4}\left(\rho_{0} \frac{v_{0}^{2}}{\varepsilon_{D}}\right)
\end{aligned}
$$

Where $\mathrm{D}_{\mathrm{C}}$ is the diameter of the volume crushed zone, $\boldsymbol{\varepsilon}_{\boldsymbol{c r d f}}$ are the length of the damage, $\boldsymbol{\rho}_{\mathbf{0}}$ is the initial density of the graded cellular core and $\boldsymbol{\rho}_{\boldsymbol{s}}$ is the density of the densified material, $\boldsymbol{v}_{\mathbf{0}}$ is the impact velocity of the projectile and $\boldsymbol{\varepsilon}_{\boldsymbol{D}}$ is the locking strain, $\boldsymbol{\sigma}_{\boldsymbol{p l}}$ is the quasi-static plateau stress and $\mathrm{K}$ is a constant factor for core crushing.

It shows that two kinds of gradient in the graded cellular material could be involved. One is the gradient in terms of the quasi-static stress plateau, while the other is in the initial density. For the present study, only gradient in the plateau stress is considered in the graded cellular core while the density is uniform along the graded cellular material. According to work reported by Chen et al. [14], a constant gradient in the quasi-static plateau stress, g, is considered in the cellular material and it can be defined as,

$$
g=\frac{\Delta \sigma}{\sigma_{0} l_{0}}
$$

Where $\Delta \boldsymbol{\sigma}$ is the difference in the quasi-static plateau stress between the two ends of the cellular core with length $\boldsymbol{l}_{\mathbf{0}}$ and $\boldsymbol{\sigma}_{\mathbf{0}}$ the average plateau stress over the graded cellular core. Appling this constant gradient to the graded sandwich with cellular core taken as reference A-4321 where the gradient is linear decreases, i.e $\mathrm{g}<0$. To do this, only shock front traveling from the strongest part to the weakest part is considered, the plateau stress can be obtained in terms of the gradient $\mathrm{g}$ and the length of the densified zone $\mathrm{z}$,

The impact piercing force model can be then written as:

$$
\sigma_{p l}=\sigma_{0}-\left(\frac{l_{0}}{2}-\frac{z}{1-\varepsilon_{D}}\right) g \sigma_{0}
$$

$$
\begin{aligned}
F_{P}= & \sqrt{2} A \pi D_{C}\left(\varepsilon_{c r d f}\right)^{1.5}+\pi D_{C} \times 550.8 \times 10^{3}\left(\frac{\rho_{0}}{\rho_{s}}\right)^{1.8}+\pi \frac{D_{C}^{2}}{4} K\left(\sigma_{0}-\left(\frac{l_{0}}{2}-\frac{z}{1-\varepsilon_{D}}\right) g \sigma_{0}\right) \\
& +\pi \frac{D_{C}^{2}}{4}\left(\rho_{0} \frac{v_{0}^{2}}{\varepsilon_{D}}\right)
\end{aligned}
$$

This model is compared with the corresponding results from FE simulation and the experimental results as shown in Figure 5. The results shows that the analytical model predict well the top skin peak load in comparison with the FE simulation and experimental results. However, it overestimate the prediction of the level piercing force of the graded cellular core. 


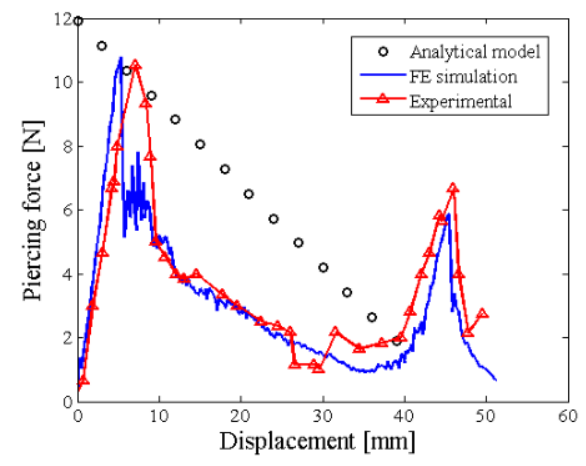

Fig. 5. Plot of piercing force-displacement traces. Comparison between the three results.

\section{Conclusion}

From the results and discussion, the following conclusions can be drawn:

(a) The numerical approach predicts with a high degree of accuracy the impact piercing force-displacement traces in the experimental test of graded core sandwich panels with decreasing gradient profiles (A-4321).

(b) The piercing force-displacement response of graded core sandwich panels is affected by the core density gradient profile.

(c) The energy absorption capability of graded core sandwich panels can be effectively enhanced by modifying the arrangement of the core layers, but only under unclumping boundary conditions, which is hard to achieve with clumping boundary conditions.

(d) An analytical model is developed to predict the top skin piercing peak load of the graded sandwich panels.

\section{References}

1. S. Venkataraman, B.V. Sankar, AIAA. 41 (2003).

2. S. Suresh, A. Mortensen, Fundamentals of Functionally Graded Materials (1998).

3. E. Etemadi, A.A. Khatibi, M. Takaffoli, Compos Struct. 89 (2009).

4. J. Zhou, Z. W. Guan, W. J. Cantwell, Eng Struct. 97 (2013).

5. J. Jing, F. Yang, L. Zhao, Eng Struct. 171 (2017).

6. G. Sun, E. Wang, H. Wanf, et al. Mater Des. (160) 2018).

7. B. O. Baba, Eng Struct. 159 (2017).

8. H. B. Zeng, S. Pattofatto, H. Zhao, et al. Int J Impact Eng. 37 (2010).

9. J. Lemaitre J. A course on damage mechanics ( $2^{\text {nd }}$ ed. Berlin: Springer; 1996)

10. S. Pattofatto, H. B. Zeng, H. Zhao. J Sandw Struct Mater. 14 (2011).

11. H. B. Zeng, S. Pattofatto, H Zhao, et al. Int J Mech Sci. 52 (2010).

12. S. R. Reid, C. Peng. Int. J. Impact. Eng. 19 (1997).

13. I. Elnasri I, H. Zhao H. Int J Impact Eng. 96 (2016).

14. C. Chen, T. Yu, G. Lu. Int J solids Struct. 50 (2013). 